\title{
Prevalência e fatores associados à capacidade funcional de idosos na Estratégia Saúde da Família em Montes Claros, Minas Gerais, Brasil
}

\author{
Prevalence and factors associated with the functional capacity of the elderly within the Family \\ Health Strategy in the city of Montes Claros, Minas Gerais, Brazil
}

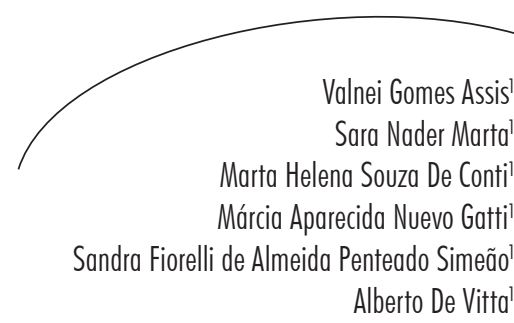

Resumo

Objetivou-se mensurar o nível de capacidade funcional de idosos e suas associações, por meio de um estudo transversal com 516 residentes nas áreas circunscritas à Estratégia Saúde da Família no distrito de Grande Santos Reis, Montes Claros-MG. Foram aplicados dois questionários: um de características sociodemográficas, condições de moradia e percepção da própria situação econômica, e outro para a avaliação da capacidade funcional. Foram realizadas abordagens descritiva (frequências absoluta e relativa para variáveis categóricas, média e desvio-padrão para as contínuas) e analítica (teste de associação do qui-quadrado e regressão logística binária hierarquizada). Do total

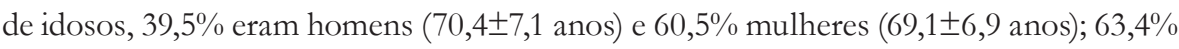
com escolaridade de zero a dois anos; $85,7 \%$ tinham renda de até dois salários mínimos; $73,1 \%$ moravam com seus familiares; $33,9 \%$ apresentaram capacidade funcional não adequada, sendo $38,5 \%$ mulheres e $27,0 \%$ homens. A capacidade funcional não adequada foi associada às mulheres, ter mais que 70 anos de idade, renda de até dois salários mínimos e menor tempo de escolaridade. Há necessidade de direcionar a atenção à saúde do idoso, aliando o incentivo à pesquisa e às ações planejadas e direcionadas para melhorar as condições de saúde e qualidade de vida desse grupo.

\section{Abstract}

This paper aimed to assess the functional capacity level of elderly people of the Family Health Strategy (FHS) and its associations. A cross-sectional study with 516 elderly residents living in the FHS surrounding area, in the district of Grande Santos Reis, Montes Claros city, Minas Gerais state, Brazil. Two questionnaires were applied: one of socio-demographic characteristics, living conditions and awareness of own economic situation; and the other assessed functional capacity. We performed descriptive (absolute and relative frequencies for categorical variables and mean and standard deviation) and analytical (test of association chi-square and binary logistic

Palavras-chave: Estratégia Saúde da Família. Idoso. Envelhecimento. Fatores de Risco. Capacidade Funcional.
Key words: Family Health Strategy. Elderly. Aging. Risk Factors. Health of the Elderly. 
regression hierarchical) approaches. Of the total, $39.5 \%$ were men (70.4 \pm 7.1 years) and $60.5 \%$ women $(69.1 \pm 6.9$ years); $63.4 \%$ with schooling from zero to two years; $85.7 \%$ had income up two minimum wages; $73.1 \%$ lived with their families; $33.9 \%$ presented inadequate functional capacity (38.5\% women and $27.0 \%$ men). The functional capacity of inadequacy was associated with women, having more than 70 years, income of up to two minimum wages and less schooling time. There is a need to direct attention to the health of the elderly, combining the promotion of research and actions planned and directed to improving the health and quality of life of this group.

\section{INTRODUÇÃO}

O envelhecimento é entendido como um processo que provoca alterações em vários sistemas corporais, acarretando limitações visuais, auditivas, motoras e intelectuais, além de um conjunto de doenças crônicas. Essas disfunções levam à diminuição da saúde geral e, em diversos graus, à redução da capacidade funcional, desencadeando a dependência nas atividades cotidianas. ${ }^{1}$

A capacidade funcional pode ser definida como "um construto que indica o máximo possível de funcionalidade que uma pessoa pode atingir em um dado momento". Para os idosos, esta avaliação é relacionada aos aspectos práticos das atividades de cuidado pessoal e ao grau de manutenção da capacidade, para o desempenho das atividades básicas e mais complexas do cotidiano. $^{2}$

Para avaliar a capacidade funcional, os métodos habitualmente adotados consistem na observação direta (testes de desempenho) e por meio de escalas que aferem os principais componentes da capacidade funcional, como Medical Outcomes Stewart Study Physical Functioning Measure (MOS), ${ }^{3}$ Functional Activities Questionnaire (FAQ), ${ }^{3}$ Functional Hamilton Independence Measure $(\mathrm{FIM}),{ }^{4}$ Escala de Avaliação de Capacidade Funcional de Rikli \& Jones ${ }^{5}$ e outros. $^{6}$

Alguns estudos, utilizando a escala de Rikli \& Jones, ${ }^{5}$ relatam a prevalência de baixa capacidade funcional em idosos. ${ }^{7,8} \mathrm{Em}$ uma cidade do nordeste brasileiro, observou-se que $21,1 \%$ dos idosos apresentaram baixa capacidade funcional, tendo os homens índices mais elevados.
No Sul do Brasil, notou-se que 37,1\% dos idosos apresentaram capacidade funcional não adequada e houve associação com a idade, com o sexo feminino, com situação econômica razoável, ruim ou muito ruim e baixa renda familiar. ${ }^{8}$

A avaliação da capacidade funcional constitui uma ferramenta simples e útil que fornece informações sobre o perfil do idoso, identificação das limitações e perda da sua autonomia. Além disso, permite definir estratégias de promoção de saúde aos idosos, visando retardar ou prevenir as incapacidades ${ }^{9} \mathrm{e}$, por conseguinte, diminuir os impactos que os tratamentos geram no orçamento das políticas públicas para a assistência à saúde. ${ }^{10}$

Nota-se que os estudos realizados refletem o perfil da população de grandes centros, envolvendo populações com costumes e características diferentes da população dos pequenos municípios. ${ }^{11}$ Dessa forma, a proposta desta pesquisa foi avaliar o nível de capacidade funcional de idosos cadastrados na Estratégia Saúde da Família (ESF) e suas associações com as características sociodemográficas, condições de moradia e autopercepção da situação econômica, na cidade de Montes Claros, região norte do estado de Minas Gerais, Brasil.

\section{MATERIAL E MÉTODOS}

Realizou-se estudo transversal com idosos residentes nas áreas circunscritas à Estratégia Saúde da Família (ESF) no distrito de Grande Santos Reis, no município de Montes Claros. Este é atendido por 11 equipes de ESF, sendo seis no próprio distrito: Santos Reis, Jardim Amazonas, Bela Vista, Bela Paisagem, Vila São Francisco de Assis e Vila Atlântida. 
A população estimada para o ano de 2010, em Montes Claros foi de 352.384 habitantes, dos quais $9,4 \%$ eram pessoas com 60 ou mais anos de idade.

O Distrito de Grande Santos Reis é composto por 21 bairros, possuindo 38.419 habitantes, dos quais $3,5 \%$ são idosos..$^{12} \mathrm{~A}$ maioria das moradias é de alvenaria, conta com fornecimento de energia elétrica, abastecimento de água tratada, coleta de lixo, e quase toda a área é beneficiada com sistema de esgoto sanitário público.

O tamanho amostral para avaliar a prevalência da incapacidade funcional foi calculado a partir da população de 1.300 idosos do distrito. Para esta análise, adotou-se a prevalência estimada de $29 \%$, erro aceitável de três pontos percentuais, nível de confiança de $95 \%$, efeito de delineamento de 1,5. Considerando tais prerrogativas, estimouse um total de 516 idosos.

O estudo foi realizado a partir de uma amostra por conglomerados em dois estágios: no primeiro, as equipes da ESF foram selecionadas como unidades básicas de seleção. Nestas unidades, foi realizada a amostragem estratificada proporcional à quantidade de idosos cadastrados por área de abrangência de cada agente de saúde e ao gênero. No segundo estágio, o idoso foi considerado a unidade amostral, também sendo escolhido randomicamente com base no cadastro das famílias atendidas pelo agente de saúde.

Os agentes comunitários de saúde forneceram listas com os nomes (cada nome recebeu uma numeração) e endereços de todos os idosos cadastrados nas ESF do bairro e, a partir dessa listagem, realizou-se um sorteio para selecionar os participantes do estudo. Utilizaram-se como critérios de inclusão pessoas com idade igual ou superior a 60 anos, de ambos os sexos e assistidas por uma das ESF selecionadas. Foram excluídas as pessoas incapazes de se comunicar e os que se recusaram a participar ou a assinar o Termo de Consentimento Livre e Esclarecido (TCLE). No caso de domicílio fechado após três tentativas ou impossibilidade de resposta ao questionário, foi sorteado outro idoso da lista.

\section{Procedimento de coleta de dados}

Após autorização da Secretaria Municipal de Saúde, os idosos foram contatados, esclarecidos a respeito dos objetivos da pesquisa e convidados a participar. Foi garantido, pelos pesquisadores, o caráter voluntário da participação, o sigilo e o anonimato dos dados obtidos nas entrevistas e a possibilidade de abandonar a pesquisa a qualquer momento. Ao concordarem, assinaram o Termo de Consentimento Livre e Esclarecido (TCLE). A pesquisa teve a aprovação do Comitê de Ética em Pesquisa da Universidade Sagrado Coração, através do parecer no 163/2009.

Considerando a multiplicidade das variáveis investigadas, o amplo universo de sujeitos, o instrumento de investigação e as características da equipe, foi realizado estudo piloto com 30 idosos pertencentes a uma equipe da ESF do distrito, com a finalidade de averiguar a exequibilidade do projeto, evidenciando as dificuldades para resolvê-las e minimizálas, assim como promover o treinamento dos entrevistadores, reduzindo a possibilidade de tendenciosidade na coleta de dados.

As entrevistas foram realizadas por seis agentes comunitários de saúde e supervisionadas pelo pesquisador. Os entrevistadores foram treinados com base em um protocolo de padronização dos procedimentos de coleta de dados (teórico e prático), previamente estabelecido no sentido de minimizar os possíveis erros intra e interavaliadores. O supervisor também realizou o controle de qualidade, que consistiu na aplicação de questionários com número reduzido de questões a $10 \%$ dos entrevistados.

O período para a realização das entrevistas foi de fevereiro a setembro de 2010 nas residências dos idosos, levando em média 50 minutos. Foram aplicados dois questionários: um de características sociodemográficas, condições de moradia e percepção da própria situação econômica ${ }^{8}$ e outro de autoavaliação da capacidade funcional proposto por Rikli \& Jones. ${ }^{5}$

No primeiro, as variáveis consideradas foram: (1) sexo; (2) idade; (3) tipo de moradia; (4) com 
quem mora; (5) como considera sua residência; (6) qual a renda aproximada; (7) soma dos recursos de todos os moradores em salários mínimos; (8) quantas pessoas colaboram com esta renda; (9) quantas pessoas usufruem desta renda; (10) alguém fora de casa contribui para cobrir suas despesas; (11) quantas pessoas fora de casa dependem financeiramente do(a) $\operatorname{sr}(a)$; (12) como avalia sua situação econômica para satisfazer suas necessidades básicas; comparando com outras pessoas da sua idade sua situação econômica parece ser; e (13) quantos anos de escolaridade completos cursou. ${ }^{8}$

O segundo instrumento foi escolhido considerando-se que foi utilizado por outros estudos nacionais e a possibilidade de incluir todos os idosos fisicamente dependentes $\mathrm{e}$ independentes, permitindo ampliar a extensão da avaliação com a coleta de dados da distribuição dos problemas funcionais na população. ${ }^{6,8}$

Esta escala é composta por 12 atividades: a) tomar conta de suas necessidades pessoais, como vestir-se; b) tomar banho, usar o banheiro; c) caminhar fora de casa (um a dois quarteirões); d) fazer atividades domésticas leves como cozinhar, tirar o pó, lavar pratos, varrer ou andar de um lado para o outro na casa; e) subir ou descer escadas; f) fazer compras no supermercado ou no centro; g) levantar e carregar $5 \mathrm{~kg}$ (como por exemplo, um pacote de arroz); h) caminhar seis a sete quarteirões; i) caminhar 12 a 14 quarteirões; j) levantar e carregar $13 \mathrm{~kg}$ de peso (como uma mala média grande); k) fazer atividade doméstica pesada, como aspirar, esfregar pisos, passar o rastelo; l) fazer atividades vigorosas como andar grandes distâncias, cavoucar o jardim, mover objetos pesados, atividades de dança aeróbica ou ginástica vigorosa. Para cada atividade existem três opções de resposta, de acordo com a habilidade do indivíduo em realizá-las: 1) faço; 2) faço com ajuda ou com dificuldade; e 3) não posso fazer. Estes valores são somados e resultam num escore final.

A capacidade funcional foi dicotomizada em "adequada" e "não adequada". Foram considerados como capacidade funcional adequada os níveis funcionais avançados (realiza 12 itens sem dificuldade) e moderado (realiza 7 a 11 itens sem dificuldade). Como capacidade funcional não adequada, os indivíduos que apresentaram dificuldade ou necessitaram de ajuda na realização de seis ou mais atividades, ou ainda, não tiveram condições de realizar três delas. ${ }^{8}$

\section{Procedimento de análise de dados}

Os dados obtidos foram introduzidos em um banco de dados e submetidos ao programa estatístico SPSS, versão 10.0. A análise foi realizada mediante abordagens descritiva e analítica. $\mathrm{Na}$ descritiva, foram feitas as distribuições de frequências absoluta e relativa para variáveis categóricas, e média e desviopadrão para as variáveis contínuas. Na analítica, utilizaram-se a técnica bivariada do teste de associação do qui-quadrado e o teste exato de Fisher e, em seguida, a análise multivariada por regressão logística binária hierarquizada. ${ }^{13}$ Os fatores investigados foram agrupados em blocos, ordenados de acordo com a precedência com que atuariam sobre a capacidade funcional do idoso (bloco socioeconômico, demográfico, condições de moradia e percepção da situação econômica). ${ }^{13}$ Para a seleção das variáveis destinadas à modelagem, adotou-se $\mathrm{p}<0,20$ como nível crítico. As variáveis dos blocos mais distais, permaneceram como fatores de ajuste para os blocos hierarquicamente inferiores. As variáveis selecionadas foram mantidas no modelo, mesmo que sua significância estatística não fosse preservada com a inclusão de blocos hierárquicos inferiores. ${ }^{13}$ Considerou-se um nível de significância de 5\% e intervalo de confiança (IC) de 95\%, com cálculo dos odds ratios ajustados. ${ }^{14}$

\section{RESULTADOS}

Foram convidados a participar do estudo 558 idosos, dos quais $28(5,0 \%)$ não preencheram 
os critérios de inclusão e $14(2,5 \%)$ recusaram. O total de participantes, portanto, foi de 516 idosos, sendo 204 do sexo masculino, com média de idade de 70,4 $\pm 7,1$ anos, e 312 do feminino, com média de idade de 69,1 16,9 anos. Quanto aos anos de escolaridade, 63,4\% possuíam de zero a dois anos, com renda de até dois salários mínimos para $85,7 \%$ dos entrevistados (tabela 1 ).

Tabela 1. Distribuição de frequências absoluta e relativa das características sociodemográficas e percepção da própria situação econômica dos idosos. Montes Claros-MG, 2010.

\begin{tabular}{|c|c|c|c|}
\hline \multirow{2}{*}{ Variáveis } & \multicolumn{2}{|c|}{ Sexo } & \multirow{2}{*}{$\begin{array}{c}\text { Total } \\
\mathrm{n}=516\end{array}$} \\
\hline & $M(n=204)$ & $\mathrm{F}(\mathrm{n}=312)$ & \\
\hline \multicolumn{4}{|l|}{ Faixa etária } \\
\hline 60 a 69 anos & $105(51,5 \%)$ & $198(63,5 \%)$ & $303(58,7 \%)$ \\
\hline 70 anos ou mais & $99(48,5 \%)$ & $114(36,5 \%)$ & $213(41,3 \%)$ \\
\hline \multicolumn{4}{|l|}{ Anos de escolaridade } \\
\hline De zero a dois anos & $121(59,3 \%)$ & $206(66,0 \%)$ & $327(63,4 \%)$ \\
\hline De três a cinco anos & $83(40,7 \%)$ & $106(34,0 \%)$ & $189(36,6 \%)$ \\
\hline \multicolumn{4}{|l|}{ Renda } \\
\hline Até dois SMs & $170(83,3 \%)$ & $272(87,2 \%)$ & $442(85,7 \%)$ \\
\hline Dois a quatro $\mathrm{SMs}$ & $34(16,7 \%)$ & $40(12,8 \%)$ & $74(14,3 \%)$ \\
\hline \multicolumn{4}{|c|}{$\begin{array}{l}\text { Quantas pessoas (moradores) colaboram } \\
\text { com esta renda }\end{array}$} \\
\hline Até duas & $73(35,8 \%)$ & $151(48,4 \%)$ & $224(43,4 \%)$ \\
\hline Acima de três & $131(64,2 \%)$ & $161(51,6 \%)$ & $292(56,6 \%)$ \\
\hline \multicolumn{4}{|c|}{ Quantas pessoas usufruem desta renda } \\
\hline Uma & $15(7,4 \%)$ & $30(9,6 \%)$ & $45(8,7 \%)$ \\
\hline Duas ou mais & $189(92,6 \%)$ & $282(90,4 \%)$ & $471(91,3 \%)$ \\
\hline \multicolumn{4}{|c|}{$\begin{array}{l}\text { Alguém contribui para cobrir suas } \\
\text { despesas? (fora de casa) }\end{array}$} \\
\hline Sim & $176(86,3 \%)$ & $266(85,3 \%)$ & $442(85,7 \%)$ \\
\hline Não & $28(13,7 \%)$ & $46(14,7 \%)$ & $74(14,3 \%)$ \\
\hline \multicolumn{4}{|l|}{ Situação econômica atual } \\
\hline Muito boa/boa & $51(25,0 \%)$ & $85(27,2 \%)$ & $136(26,4 \%)$ \\
\hline Razoável/ruim & $153(75,0 \%)$ & $227(72,8 \%)$ & $380(73,6 \%)$ \\
\hline \multicolumn{4}{|c|}{$\begin{array}{l}\text { Situação econômica comparada a outra } \\
\text { pessoa }\end{array}$} \\
\hline Melhor & $74(42,3 \%)$ & $86(25,2 \%)$ & $160(31,0 \%)$ \\
\hline Igual ou pior & $101(57,7 \%)$ & $255(74,8 \%)$ & $356(69,0 \%)$ \\
\hline
\end{tabular}

$\mathrm{SM}=$ salário mínimo. 
Observou-se que os idosos caracterizaramse por morar em casa própria $(96,1 \%)$, com seus familiares $(73,1 \%)$, consideraram sua residência ruim $(96,1 \%)$ e classificaram sua situação econômica como razoável ou ruim $(73,6 \%)$ (tabela 2).
Dos idosos entrevistados, $33,9 \% \quad(175)$ apresentaram capacidade funcional não adequada, dos quais $68,5 \%$ (120) eram mulheres e $31,5 \%$ (55), homens.

Tabela 2. Distribuição de frequências absoluta e relativa das condições de moradia e capacidade funcional dos idosos. Montes Claros-MG, 2010.

\begin{tabular}{lccc}
\hline \multirow{2}{*}{ Variáveis } & \multicolumn{2}{c}{ Sexo } & Total \\
\cline { 2 - 3 } Moradia & $\mathrm{M}(\mathrm{n}=204)$ & $\mathrm{F}(\mathrm{n}=312)$ & $\mathrm{n}=516$ \\
\hline Própria & $198(97,1 \%)$ & $298(95,5 \%)$ & $496(96,1 \%)$ \\
Alugada & $6(2,9 \%)$ & $14(4,5 \%)$ & $20(3,9 \%)$ \\
\hline Mora com quem & & & \\
Sozinho(a) & $44(21,6 \%)$ & $95(30,4 \%)$ & $139(26,9 \%)$ \\
Esposo(a) e filho(s) & $160(78,4 \%)$ & $217(69,6 \%)$ & $377(73,1 \%)$ \\
\hline Como mora & & $303(97,1 \%)$ & $496(96,1 \%)$ \\
Muito ruim/ruim & $193(94,6 \%)$ & $9(2,9 \%)$ & $20(3,9 \%)$ \\
Boa/muito boa & $11(5,4 \%)$ & $192(61,5 \%)$ & $341(66,1 \%)$ \\
\hline Capacidade funcional & $149(73,0 \%)$ & $175(33,9 \%)$ \\
Adequada & $55(27,0 \%)$ & $120(38,5 \%)$ & \\
Não adequada & & & \\
\hline
\end{tabular}

A análise bivariada (tabela 3) mostrou uma diminuição da capacidade funcional $(\mathrm{p}<0,05)$ quando associada ao aumento da idade, em mulheres, em idosos com menor tempo de escolaridade, renda até dois salários mínimos, que moravam com esposo(a) e filho(s) e com situação econômica atual razoável. 
Tabela 3. Análise bivariada da capacidade funcional com as variáveis sociodemográficas, condições de moradia dos idosos e percepção da própria situação econômica. Montes Claros-MG, 2010.

\begin{tabular}{|c|c|c|c|}
\hline \multirow{2}{*}{ Variáveis } & \multicolumn{2}{|c|}{ Capacidade Funcional } & \multirow{2}{*}{ Valor de $\mathrm{p}$} \\
\hline & Não Adequada & Adequada & \\
\hline \multicolumn{4}{|l|}{ Sexo } \\
\hline Masculino & $55(31,4 \%)$ & $149(43,7 \%)$ & \multirow{2}{*}{0,00697} \\
\hline Feminino & $120(68,6 \%)$ & $192(56,3 \%)$ & \\
\hline \multicolumn{4}{|l|}{ Faixa etária } \\
\hline 60 a 69 anos & $72(41,1 \%)$ & $231(67,7 \%)$ & \multirow{2}{*}{0,00000001} \\
\hline 70 anos ou mais & $103(58,9 \%)$ & $110(32,3 \%)$ & \\
\hline \multicolumn{4}{|l|}{ Anos de escolaridade } \\
\hline De zero a dois anos & $113(64,6 \%)$ & $159(46,6 \%)$ & \multirow{2}{*}{0,00011} \\
\hline De três a cinco anos & $62(35,4 \%)$ & $182(53,4 \%)$ & \\
\hline \multicolumn{4}{|l|}{ Moradia } \\
\hline Própria & $166(94,9 \%)$ & $330(96,8 \%)$ & \multirow{2}{*}{0,28549} \\
\hline Alugada & $9(5,1 \%)$ & $11(3,2 \%)$ & \\
\hline \multicolumn{4}{|l|}{ Mora com quem } \\
\hline Sozinho(a) & $61(34,9 \%)$ & $78(22,9 \%)$ & \multirow{2}{*}{0,00367} \\
\hline Esposo e filho(s) & $114(65,1 \%)$ & $263(77,1 \%)$ & \\
\hline \multicolumn{4}{|l|}{ Como mora } \\
\hline Muito ruim/ruim & $171(97,7 \%)$ & $325(95,3 \%)$ & \multirow{2}{*}{0,18002} \\
\hline Boa/muito boa & $4(2,3 \%)$ & $16(4,7 \%)$ & \\
\hline \multicolumn{4}{|l|}{ Renda } \\
\hline Até dois SMs & $159(90,9 \%)$ & $283(83,0 \%)$ & \multirow{2}{*}{0,01580} \\
\hline De dois a quatro SMs & $16(9,1 \%)$ & $58(17,0 \%)$ & \\
\hline \multicolumn{4}{|c|}{ Quantas pessoas colaboram com renda } \\
\hline Até duas & $86(49,1 \%)$ & $138(40,5 \%)$ & \multirow{2}{*}{0,05984} \\
\hline Acima de três & $89(50,9 \%)$ & $203(59,5 \%)$ & \\
\hline \multicolumn{4}{|c|}{ Quantas pessoas usufruem desta renda } \\
\hline Uma & $17(9,7 \%)$ & $28(8,2 \%)$ & \multirow{2}{*}{0,56669} \\
\hline Duas ou mais & $158(90,3 \%)$ & $313(91,8 \%)$ & \\
\hline \multicolumn{4}{|c|}{$\begin{array}{l}\text { Alguém contribui para cobrir suas despesas? } \\
\text { (fora de casa) }\end{array}$} \\
\hline $\operatorname{Sim}$ & $152(86,9 \%)$ & $290(85,0 \%)$ & \multirow{2}{*}{0,57799} \\
\hline Não & $23(13,1 \%)$ & $51(15,0 \%)$ & \\
\hline \multicolumn{4}{|l|}{ Situação econômica atual } \\
\hline Muito boa/boa & $49(28,0 \%)$ & $87(25,5 \%)$ & \multirow{2}{*}{0,54384} \\
\hline Razoável/ruim & $126(72,0 \%)$ & $254(74,5 \%)$ & \\
\hline \multicolumn{4}{|c|}{$\begin{array}{l}\text { Situação econômica comparada a } \\
\text { outra pessoa }\end{array}$} \\
\hline Melhor & $74(42,3 \%)$ & $86(25,2 \%)$ & \multirow{2}{*}{0,00007} \\
\hline Igual ou pior & $101(57,7 \%)$ & $255(74,8 \%)$ & \\
\hline
\end{tabular}


Os resultados da análise de regressão logística (tabela 4) evidenciaram que idade, escolaridade, renda e sexo mostraram associação de forma independente com a baixa capacidade funcional.

Tabela 4. Análise multivariada de regressão logística, modelo final, para associações independentes com a capacidade funcional não adequada. Montes Claros-MG, 2010.

\begin{tabular}{|c|c|c|c|c|c|}
\hline Variáveis & $\begin{array}{l}\text { OR bruto/ } \\
\text { IC } 95 \%\end{array}$ & p bruto & $\begin{array}{c}\text { OR ajustado/ IC } \\
95 \%\end{array}$ & $\mathrm{RP}$ & p ajustado \\
\hline \multicolumn{6}{|l|}{ Sexo } \\
\hline Masculino & 1,00 & \multirow{2}{*}{0,002} & 1,00 & \multirow{2}{*}{1,46} & \multirow{2}{*}{0,002} \\
\hline Feminino & $1,69(1,15-2,48)$ & & $1,92(1,26-2,91)$ & & \\
\hline \multicolumn{6}{|l|}{ Faixa etária } \\
\hline 60 a 69 anos & 1,00 & \multirow{2}{*}{0,003} & 1,00 & \multirow{2}{*}{2,04} & \multirow[t]{2}{*}{0,000} \\
\hline 70 anos ou mais & $3,00(2,06-4,38)$ & & $2,84(1,89-4,26)$ & & \\
\hline \multicolumn{6}{|c|}{ Anos de escolaridade } \\
\hline De 3 a 5 anos & 1,00 & \multirow{2}{*}{0,000} & 1,00 & \multirow{2}{*}{1,63} & \multirow[t]{2}{*}{0,002} \\
\hline De 0 a 2 anos & $1,35(1,18-1,54)$ & & $1,24(1,08-1,43)$ & & \\
\hline \multicolumn{6}{|l|}{ Renda } \\
\hline De 2 a 4 SMs & 1,00 & \multirow{2}{*}{0,02} & 1,00 & \multirow[t]{2}{*}{1,66} & \multirow[t]{2}{*}{0,04} \\
\hline Até $2 \mathrm{SMs}$ & $1,39(1,04-1,85)$ & & $1,35(1,00-1,83)$ & & \\
\hline \multicolumn{6}{|c|}{ Situação econômica atual } \\
\hline Muito boa/boa & 1,00 & \multirow{2}{*}{0,01} & 1,00 & \multirow[t]{2}{*}{1,09} & \multirow[t]{2}{*}{ * } \\
\hline Razoável/ruim & $1,44(1,08-1,93)$ & & $*$ & & \\
\hline
\end{tabular}

$\mathrm{SM}=$ salário mínimo; *excluído após ajuste por idade, sexo e escolaridade.

\section{DISCUSSÃO}

As características da amostra estudada em relação à capacidade funcional não adequada $(33,9 \%)$ coincidem com os resultados encontrados no Ceará $(21,1 \%)^{7}$ e em Santa Catarina $(37,1 \%),{ }^{8}$ que utilizaram a mesma escala.

No presente estudo, a análise multivariada mostrou que houve associação entre o sexo, faixa etária, anos de escolaridade, renda e situação econômica atual com a capacidade funcional não adequada.

Os idosos com 70 anos ou mais apresentaram $28 \%$ mais chances de capacidade funcional não adequada. Associando as distintas faixas etárias, observou-se que o aumento da idade implica maior comprometimento da capacidade funcional. De acordo com alguns autores, idosos acima de 70 anos têm 2,8 vezes mais chances de apresentar comprometimento da capacidade funcional, e de 85 anos e mais apresentaram 2,9 vezes mais chances em relação aos idosos abaixo de 85 anos. $^{15,16}$

O avanço da idade é considerado um dos mais importantes fatores de risco para a deterioração da capacidade funcional dos idosos, devido ao aumento das alterações fisiológicas (nos sistemas sensorial, neurológico e musculoesqueléticos) e da prevalência de doenças crônicas. ${ }^{17,18}$ 
As mulheres entrevistadas apresentaram maior prevalência de capacidade funcional não adequada (68,5\%), confirmando os achados de outros autores. ${ }^{15,19-22}$ As mulheres, que são maioria em grupo de idosos, apesar de terem maior expectativa de vida, apresentam maiores limitações ou maior perda da capacidade funcional. Algumas hipóteses são levantadas para explicar essa diferença: maior prevalência de condições incapacitantes não fatais (osteoporose, osteoartrite e depressão, por exemplo) e maior habilidade da mulher em reportar suas condições de saúde em relação aos homens da mesma faixa etária e pelas diferenças na sobrevivência e nas morbidades associadas. . $^{15,23,24}$

Os idosos entrevistados com menor quantidade de anos de estudo e que possuíam renda até dois salários mínimos apresentaram maior percentual de capacidade funcional não adequada. Os relacionamentos entre estas mesmas variáveis são apontados em estudos brasileiros utilizando dados da Pesquisa Nacional por Amostras de Domicílios (PNAD), que têm mostrado que a capacidade funcional dos idosos é fortemente influenciada pela renda domiciliar per capita. Para a população idosa, a relação entre pior nível socioeconômico e saúde foi mais visível em relação aos indicadores gerais das condições de saúde: pior percepção da própria saúde, incapacidade para realizar atividades devido a problemas de saúde e ter estado recentemente acamado. ${ }^{25}$

Pesquisa realizada na cidade de Ubá-MG mostrou que os idosos analfabetos e com renda per capita menor que 250 reais mostraram-se com maior chance de apresentar capacidade funcional não adequada. Dessa forma, o nível socioeconômico desse grupo parece ter importante influência nas condições de saúde, especialmente na capacidade funcional. ${ }^{23} \mathrm{~A}$ proporção de idosos com dependência no dia a dia foi significativamente mais alta entre idosos de baixa renda, ${ }^{26}$ assim como a baixa renda estava relacionada com a incapacidade funcional em dois terços dos entrevistados em sete países. ${ }^{27}$ Da mesma forma, a baixa renda per capita dos idosos, associada à baixa escolaridade, priva-os de uma adequada assistência de saúde, da compra dos medicamentos ou do pagamento da mensalidade do plano de saúde, com reflexos diretos na sua performance funcional, uma vez que a renda é um dos elementos essenciais para a preservação da autonomia e para a manutenção ou recuperação da saúde. ${ }^{11,28}$

Estudo realizado com idosos de um plano de saúde mostrou que os indivíduos com até quatro anos de escolaridade tinham prevalência de capacidade funcional não adequada três vezes mais frequente do que aqueles de escolaridade elevada. ${ }^{29}$ Resultados semelhantes foram observados no inquérito epidemiológico realizado nos Distritos Sanitários da Restinga e do Extremo-sul, em Porto Alegre. ${ }^{30}$

Oportunidades de aprendizado podem ajudar as pessoas a desenvolver as habilidades e confiança para se adaptar e desenvolver um processo de envelhecimento saudável. Idosos com melhores condições financeiras geralmente possuem melhor acesso a prevenção, tratamento e reabilitação. ${ }^{21,22,27}$

Algumas limitações deste estudo, no entanto, precisam ser relatadas. Primeiramente, optou-se por não analisar algumas variáveis comportamentais (nível de atividade física e tabagismo) e relacionadas à saúde do idoso (número e tipos de doença, saúde mental e hospitalização nos últimos seis meses) como potenciais fatores associados à incapacidade funcional. Além disso, no instrumento aplicado fica implícito que as atividades investigadas fazem parte do cotidiano do entrevistado. Embora tenha sido pouco frequente, nos casos em que essa perspectiva não se confirmou, o idoso era instigado a pensar na possibilidade de que, se necessário, ele desempenharia tal tarefa sem ajuda de outra pessoa. Também a avaliação de tal capacidade por meio de questionários é um tanto subjetiva, para quantificação de detalhes minuciosos de incapacidades, o que requer uso de instrumentos mais objetivos. 


\section{CONCLUSÃO}

Os resultados do presente estudo apontam para maior prevalência de capacidade funcional não adequada entre as mulheres, acima de 70 anos de idade, com renda de até dois salários mínimos e menor tempo de escolaridade, compatíveis com estudos nacionais e internacionais. Merecem destaque alguns importantes aspectos deste estudo: o baixo percentual de perda amostral, não havendo comprometimento da validade interna da pesquisa, o tamanho da amostra e suas características geográficas.

\section{REFERÊNCIAS}

1. Kalache A. O mundo envelhece: é imperativo criar um pacto de solidariedade social. Ciênc Saúde Coletiva 2008;13(4):1107-11.

2. Organização Mundial da Saúde, Organização Panamericana de Saúde, Centro Colaborador da OMS para Classificação de Doenças em Português. Classificação Internacional de incapacidade, funcionalidade e saúde (CIFI). São Paulo: EDUSP; 2003.

3. Stewart AL, Kamberg CJ. Physical functioning measures. In: Stewart AL, Ware Junior JE, editors. Measuring functioning and well-being: the medical outcomes study approach. [S.1.]: Rand Corporation; 1992. p. 86-101.

4. Hamilton BB, Granger C. A uniform national data system for medical rehabilitation. In: Fuhrer MJ, editor. Rehabilitation outcomes: analysis and measurement. Baltimore: Paul H. Brookes; c1987. p. 137-47.

5. Rikli RE, Jones CJ. Development and validation of a functional fitness test for community-residing older adults. J Aging Phys Act 1999;7(2):129-61.

6. Paixão Júnior $\mathrm{CM}$, Reichenheim ME. Uma revisão sobre instrumentos de avaliação do estado funcional do idoso. Cad Saúde Publica 2005;21(1):7-19.

7. Silva GX, Costa CLA, Capistrano RDS. Nível de atividade física habitual e capacidade funcional de idosos atendidos pelo Programa Saúde da Família de Juazeiro do Norte, Ceará. Efdportes.com [Internet] 2010 [citado em xx xxx xxxx];15(147):1-4. Disponível em: http://www.efdeportes.com/efd147/atividadefisica-habitual-de-idosos.htm
Diante dos resultados obtidos, há necessidade de direcionar a atenção à saúde do idoso, aliando incentivo à pesquisa, ações planejadas para melhorar as condições de saúde e qualidade de vida desse grupo. Além disso, é necessário despertar entre os governantes a luta pela diminuição do impacto da desigualdade social e pelo direito de todos ao acesso de melhores condições de vida e saúde, de modo a garantir um envelhecimento saudável. E por fim, os serviços de atenção básica devem atender adequadamente às pessoas idosas, visando à sensibilização e à educação nos cuidados primários, seguindo as necessidades específicas dessa população.

8. Fiedler MM, Peres KG. Capacidade funcional e fatores associados em idosos do sul do Brasil: um estudo de base populacional. Cad Saúde Pública 2008;24 (2):409-15.

9. Ruiz T, Monteiro A, Corrente JE, Netto MC. Avaliação do grau de satisfação dos idosos com a qualidade de vida em um pequeno município do Estado de São Paulo. Rev Aten Primária Saúde 2007;10(1):4-13.

10. Brasil. Conselho Nacional de Secretários de Saúde. Para entender o SUS. Brasília: CONASS, 2011. 291 p. (Coleção pra entender a Gestão do SUS 2011; vol. 1).

11. Maciel ACC, Guerra RO. Influência dos fatores biopsicossociais sobre a capacidade funcional de idosos residentes no nordeste do Brasil. Rev Bras Epidemiol 2007;10(2):178-89.

12. Instituto Brasileiro de Geografia e Estatística. Séries Históricas e Estatísticas. Estimativas, projeções e populações de 2010 [Internet]. Rio de Janeiro: IBGE; 2009 [acesso em 5 nov 2009]. Disponível em: http://seriesestatisticas.ibge.gov.br/lista_tema. aspx?op $=0 \& \mathrm{de}=35 \&$ no $=10$

13. Victora CG, Huttly SR, Fuchs SC, Olinto MT. The role of conceptual frameworks in epidemiological analisys: a hierarchical approach. Int J Epidemiol 1997;26(1):224-7.

14. ZAR JH. (2010). Biostatistical Analysis. 5th. ed. New Jersey: Prentice-Hall. 
15. Nunes MCR, Ribeiro RCL, Rosado LEFPL, Franceschini SC. Influência das características sociodemográficas e epidemiológicas na capacidade funcional de idosos residentes em Ubá, Minas Gerais. Rev Bras Fisioter 2009;13(5):376-82.

16. Nogueira SL, Ribeiro RCL, Rosado LEFPL, Franceschini SCC, Ribeiro AQ, Pereira ET. Fatores determinantes da capacidade funcional em idosos longevos. Rev Bras Fisioter 2010;14(4):322-9.

17. Millán-Calenti JC, Tubío J, Pita-Fernández S, González-Abraldes I, Lorenzo T, Fernández-Arruty $\mathrm{T}$, et al. Prevalence of functional disability in activities of daily living (ADL), instrumental activities of daily living (IADL) and associated factors, as predictors of morbidity and mortality. Arch of Gerontol Geriatr 2010;50(3):306-10.

18. 18- Guerra RO, Perracini MR, Fló CM, Funcionalidade e envelhecimento. In: Perracini MR, Fló CM. Funcionalidade e envelhecimento. Rio de Janeiro: Guanabara; 2009. p. 3-24. (Carvalho CRF, Tanaka C. Fisioterapia: teoria e prática).

19. Tavares DMS, Pereira GA, Iwamoto HH, Miranzzi SSC, Rodrigues LR, Machado ARM. Incapacidade funcional entre idosos residentes em um Município do interior de Minas Gerais. Texto Contexto Enferm 2007;16(1):32-9.

20. Giacomin KC, Peixoto SV, Uchoa E, Lima-Costa MF. Estudo de base populacional dos fatores associados à incapacidade funcional entre idosos na região metropolitana de Belo Horizonte, Minas Gerais, Brasil. Cad Saúde Pública 2008;24(6):1260-70.

21. Santos KA, koszuoski R, Dias-da-Costa JS, Pattussi MP. Fatores associados com a incapacidade funcional em idosos do Município de Guatambu, Santa Catarina, Brasil. Cad Saúde Pública 2007;23(11):2781-8.

22. Alves LC, Leite IC, Machado CJ. Fatores associados à incapacidade funcional dos idosos no Brasil: análise multinível. Rev Saúde Pública 2010;44(3):468-78.
23. Sudré MRS, Reiners AAO, Nakagawa JTT, Azevedo RCS, Floriano LA, Morita LHM. Prevalence of dependency and associated risk factors in the elderly. Acta Paul Enferm 2012;25(6):947-53.

24. Zunzunegui MV, Nunez O, Durban M, Garcia de Yébones MJ, Otero A. Decreasing prevalence of disability in activities of daily living, functional limitations and poor self-rated health: a 6 year follow-up study in Spain. Aging Clin Exp Res 2006;18(5):352-8.

25. Lima-Costa MF, Matos DL, Camargos VP, Macinko J. Tendências em dez anos das condições de saúde de idosos brasileiros: evidências da Pesquisa Nacional por Amostra de Domicílios (1998, 2003, 2008). Ciênc Saúde Coletiva 2011;16(9):3689-96.

26. Ramos LR. Fatores determinantes do envelhecimento saudável em idosos residentes em centro urbano: Projeto Epidoso, São Paulo. Cad Saúde Pública 2003;19(3):793-8.

27. Sousa RM, Ferri CP, Acosta D, Guerra M, Huang Y, Jacob KS, et al. The contribution of chronic diseases to the prevalence of dependence among older people in Latin America, China and India: a 10/66 Dementia Research Group population-based survey. BMC Geriatrics 2010:1-6.

28. Matthews RJ, Smith LK, Hancock RM, Jagger C, Spiers NA. Socioeconomic factors associated with the onset of disability in older age: a longitudinal study of people aged 75 years and over. Soc Sci Med 2005;61(7):1567-75.

29. Cardoso JH, Costa JSD. Características epidemiológicas, capacidade funcional e fatores associados em idosos de um plano de saúde. Ciênc Saúde Coletiva 2010;15(6):2871-8.

30. Pereira GN, Bastos GAN, Del Duca GF, Bós AJG. Indicadores demográficos e socioeconômicos associados à incapacidade funcional em idosos. Cad Saúde Pública 2012;28(11):2035-42. 